\title{
Dopamine transporter density of striatum showed to be increased in Schizophrenia first-episode drug-naïve patients
}

Fereshteh Sedaghat ${ }^{1}$, Styliani Spyridi ${ }^{2,3}$, Jannis Nimatoudis ${ }^{3}$, Amin Rakhshani ${ }^{4}$, Sygkliti-Herietta Pelidou ${ }^{5}$, Alexandra Papazisi ${ }^{7}$, Eleni Dedousi $^{7}$, Magdalini Tsolaki ${ }^{6}$ and Stavros J Baloyannis ${ }^{1}$

${ }^{1}$ Department of Neurology, Aristotle University Thessaloniki, S. Kiriakidi 1, Thessaloniki-Greece

${ }^{2}$ CAMEO Early Intervention Service, Cambridge and Peterborough NHS Foundation Trust, Ida Darwin, Fulbourn, Cambridge, UK

${ }^{3}$ Department of Psychiatry, Aristotle University of Thessaloniki, S. Kiriakidi, Thessaloniki-Greece

${ }^{4}$ Department of Informatics, Aristotle University Thessaloniki - Greece

${ }^{5}$ Department of Neurology, University of Ioannina, University Campus, Ioannina, Greece.

${ }^{6}$ Department of Neurology, Aristotle University of Thessaloniki, Makedonia, Greece

${ }^{7}$ Department of Nuclear Medicine, Aristotle University of Thessaloniki, S. Kiriakidi 1, Thessaloniki-Greece

\begin{abstract}
Background: Successful treatment with dopamine antagonists plays a crucial role of the neurotransmitter dopamine in the pathophysiology of schizophrenia. Little is known, however, about the dopaminergic function in schizophrenia and there is a controversy among different studies.

Methods: We evaluated the pre-synaptic dopamine transporter density with SPECT using ${ }^{123} \mathrm{I}-\mathrm{FP}-\mathrm{CIT}$ (DaT Scan), in 15 drug-naive patients $(10 \mathrm{~F}, 5 \mathrm{M})(27 \pm 3$ years old) with first-episode of schizophrenia and 20 (12 F, $8 \mathrm{M})(30 \pm 8$ years old) healthy controls. All the patients underwent MRI, perfusion SPECT using HMPAO and DaT Scan.

Results: A significant increase of striatal radiotracer uptake in DaT scan was shown in our patients with schizophrenia compared to normal group ( $\mathrm{p}=0.000$ ), while $\mathrm{rCBF}$ in these regions didn't show any significant difference. Also significant negative correlations were found between ${ }^{123} \mathrm{I}-\mathrm{FP}-\mathrm{CIT}$ binding ratios in right and left caudate nuclei and right striatum with positive symptoms of patients.

Conclusion: Elevated density of presynaptic dopamine transporters, may be part of the neuro-pathological abnormality which is associated with dopaminergic activity in schizophrenia.
\end{abstract}

\section{Introduction}

Schizophrenia, from the Greek roots skizo ("I split") and phren ("mind") is a chronic CNS disease characterized by impairment of thought process, affection, behaviour and perception. The clinical manifestations include positive symptoms such as delusions, hallucinations and thought disorganization, and negative symptoms such as lack of motivation, lack of initiative, social withdrawal, poverty of speech, extreme apathy and emotional flatness. Onset of symptoms typically occurs in young adulthood [1], with approximately $0.4-0.6 \%$ of the population affected [2,3]. Diagnosis is based on the self-reported experiences of the person, and abnormalities in behavior expressed by family members or friends, followed by a clinical assessment by a psychiatrist. No laboratory test regarding diagnosis of schizophrenia currently exists [4].

The effectiveness of schizophrenia treatment is often assessed using standardized methods, one of the most common is the Positive and Negative Syndrome Scale (PANSS). The PANSS is a 30 -item scale, which includes a 7-item positive scale, a 7-item negative scale, and a 16-item general psychopathology scale which includes ratings of mood problems. Items are rated on a 7-point scale. The PANSS is validated for schizophrenia and is based on patient and carers interview. Treatment with dopamine-antagonists suggests that dopamine plays an important role in the pathophysiology of schizophrenia $[5,6]$.

DaT Scan $\left({ }^{123} \mathrm{I}-\mathrm{FP}-\mathrm{CIT}\right)$ is a diagnostic product containing the active substance ioflupane, which is a radioiodinated analogue of cocaine. This cocaine-analogue has high affinity to dopamine transporter (DaT) located on the presynaptic axon terminals in the striatum. These terminals are projections of the dopamine neurons in the substantia nigra. Therefore, binding of DaT Scan in the striatum will reflect the number of dopaminergic neurons. It has been developed as a dopamine transporter imaging agent for single photon emission computed tomography (SPECT). This technique is sensitive enough

Correspondence to: Fereshteh Sedaghat, Marmara 21, 55132 Kalamaria, Thessaloniki, Greece; E-mail: feresh@med.auth.gr

Key words: Schizophrenia, Dopamine transporters, DaT scan, SPECT, rCBF , striatum

Received: September 22, 2017; Accepted: October 20, 2017; Published: October 23,2017 
to differentiate changes in the nigrostriatal dopaminergic system in patients with Parkinsonism and differentiate it from idiopathic tremor [7].

Drug-induced parkinsonism is one of the main causes of treatment drop-out in schizophrenia causing a high incidence of relapse that leads patients to a bad clinical prognosis which is mainly related to the blockade of striatal D2 receptor [8]. Dopaminergic nigrostriatal pathway has a role in movement control, so the study of the dopamine transporter (DaT) may be of great value to determine its effect in the appearance of Drug-induced parkinsonism [8-11].

Many neurobiological systems may be involved in the complex framework of dopaminergic dysfunction. We evaluated pre-synaptic dopamine transporter density with SPECT using ${ }^{123} \mathrm{I}$-FP-CIT (DaT Scan) and also regional cerebral blood flow rCBF using HMPAO in first episode drug-naive patients suffering from schizophrenia. Only a few imaging studies have been performed regarding the assessment of the presynaptic striatal $\mathrm{DaT}$ in acute psychotic states of schizophrenia in drug-naïve patients, and the data are not yet consistent.

\section{Methods}

Thirty-five cases, 15 drug-naive patients $(10 \mathrm{~F}, 5 \mathrm{M})(27 \pm 3$ years old), with a clinical diagnosis of schizophrenia fulfilling DSM-IV criteria and $20(12 \mathrm{~F}, 8 \mathrm{M})(30 \pm 8$ years old) normal controls are included in this study. The patients underwent general medical and neurological examination and brain magnetic resonance imaging (MRI) to rule out physical pathology and brain lesions which may produce psychotic schizophrenia-like symptoms. Our exclusion criteria included any medical or neurological conditions, any previous use of antipsychotics, lithium treatment, history of alcohol or substance dependence and head injury. Psychopathological status was assessed by Positive and Negative Syndrome Scale.

The psychopathological syndrome type was calculated following the procedure of Kay by establishing the PANSS positive-PANSS negative difference: Patients who have a syndrome type value greater than zero have positive syndrome type (presenting mainly positive symptoms of schizophrenia) and those with syndrome type value less than zero have negative syndrome type (presenting mainly negative symptoms) [12].

Brain SPECT was done after intravenously injection of $555 \mathrm{MBq}$ of

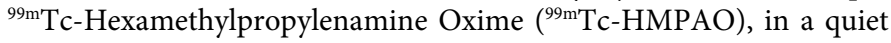
and bright room with patient's eyes open. Each patient had an IV line at least 15 minutes before the injection of the agent.

Images were acquired after 45 minutes of injection using a single headed ADAC gamma camera equipped with a low-energy-highresolution (LEHR) collimator. The patient was in a supine position with his head stabled with a special belt, if indicated. The total acquisition consisted of 120 projections acquired for 20 seconds into a $128 \times 128$-acquisition matrix. The images were processed on a Sun Pegasys computer and were reconstructed with Butterworth filter back projection. Slices were generated in parallel to the orbitomeatal line.

The attenuation correction based on Chang's method and reorientations were done on reconstructed brain images. The images were visually evaluated for their perfusion pattern and then a region of interest (ROI) accounting for blood flow (rCBF) was drawn above the whole cerebellum (CER) and ROIs with the size of $5 \times 5$ pixels were used to measure the mean count, in the following regions: right and left prefrontal cortex (RPF, LPF), frontal (RF, LF), caudate nucleus (RCN, LCN), thalamus (RTh, LTh), superior parietal (RSP,LSP), inferior parietal (RIP, LIP), medial and lateral temporal (RMT, RLT, LMT, LLT), occipital (RO, LO) and posterior cingulate gyrus (PC). The coronal slices were used to study the temporal lobes and transverse slices for studying the other regions. Semiquantitative regional cerebral blood flow (rCBF) analysis using cortex to cerebellum ratio was done. SPECT using 123I-FP-CIT followed the HMPAO scan after a time interval of up to one week. Twenty minutes before DaT Scan, all patients underwent thyroid blocking using potassium iodide to minimize radiation exposure to the thyroid gland and then underwent SPECT imaging 3-4 h after injection of $185 \mathrm{MBq}$ 123I-FP-CIT. Total acquisition took $40 \mathrm{~min}$ and consisted of 12020 -second projections into a $128 \times 128$ acquisition matrix. The images were reconstructed and evaluated visually. Then regions of interest (ROI) were selected based on Sedaghat et al. [7]. The ratios of specific to nonspecific binding for the striatum, putamen and caudate nucleus were calculated using the formula (ROI counts-occipital counts)/occipital counts. The results were compared with those of the control group.

Data were analyzed using the Statistical Package for Social Sciences (SPSS for windows, version 15). Variables were tested for normality of distribution using the Kolmogorov-Smirnov test. T test for independent samples was used to compare the mean values and Pearson correlation for evaluating different correlations.

\section{Results}

Our patients showed a negative syndrome type (Table1). Reduction of cerebral blood flow in bilateral prefrontal, occipital and right frontotemporal region, comparing to controls was observed. Blood perfusion in basal ganglia (RCN 95 $\pm 3, \mathrm{LCN} 94 \pm 4$ ) didn't differ significantly comparing to controls (RCN 94 \pm 7, LCN 95 \pm 6$)(p \leq 0.870$, $\mathrm{p} \leq 0.940$ ) (Figure 1). In DaT Scan the visually analysis of the images was interpreted as a normal image but the semiquantitave analysis of the images showed an increase in mean uptake of the radiotracer in striatum $(\mathrm{p}=0.00)$, caudate nuclei $(\mathrm{p}=0.00)$ and putamen $(\mathrm{p}=0.00)$ of patients with schizophrenia in comparison with control group (Figure 2).

We found a statistically significant negative correlation between ${ }^{123} \mathrm{I}$-FP-CIT binding ratios in right and left caudate nuclei $(\mathrm{r}=-0.838$, $\mathrm{r}=-0.824)$ and right striatum $(\mathrm{r}=-0.847)$ with positive symptoms of PANSS in our patients with schizophrenia (Pearson correlation)

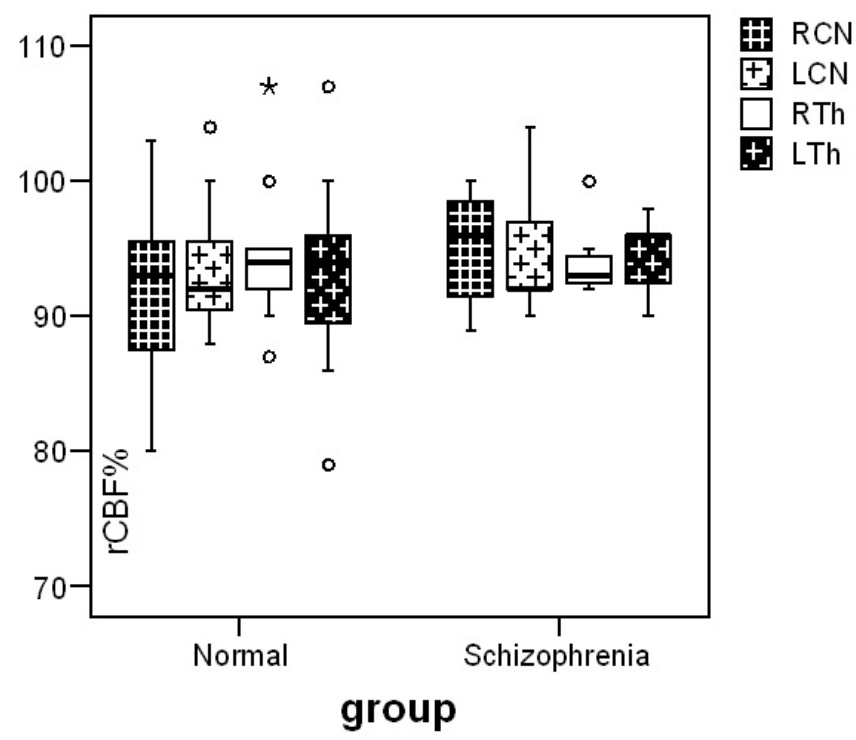

Figure 1. Median and inter-quartile range of blood flow ( $\mathrm{rCBF} \%)$ in caudate nuclei and thalamus in our two groups 
Table1. Mean PANSS scores and radiotracer uptake\% in our two groups

\begin{tabular}{|c|c|c|c|c|c|c|}
\hline Mean & Age & RST & LST & RCN & LCN & RPUT \\
\hline Normal & $30 \pm 8$ & $2.59 \pm 0.31$ & $2.47 \pm 0.32$ & $2.86 \pm 0.28$ & $2.83 \pm 0.29$ & $2.36 \pm 0.32$ \\
\hline Schizoph & $27 \pm 3$ & $3.66 \pm 0.34$ & $3.52 \pm 0.32$ & $3.75 \pm 0.30$ & $3.86 \pm 0.41$ \\
\hline PANSS & P-Neg. & P-Pos. & P-Gen. & S-Type & \\
\hline Schizoph & 21 & 17 & 37 & -3.29 & $3.34 \pm 0.38$ \\
\hline
\end{tabular}

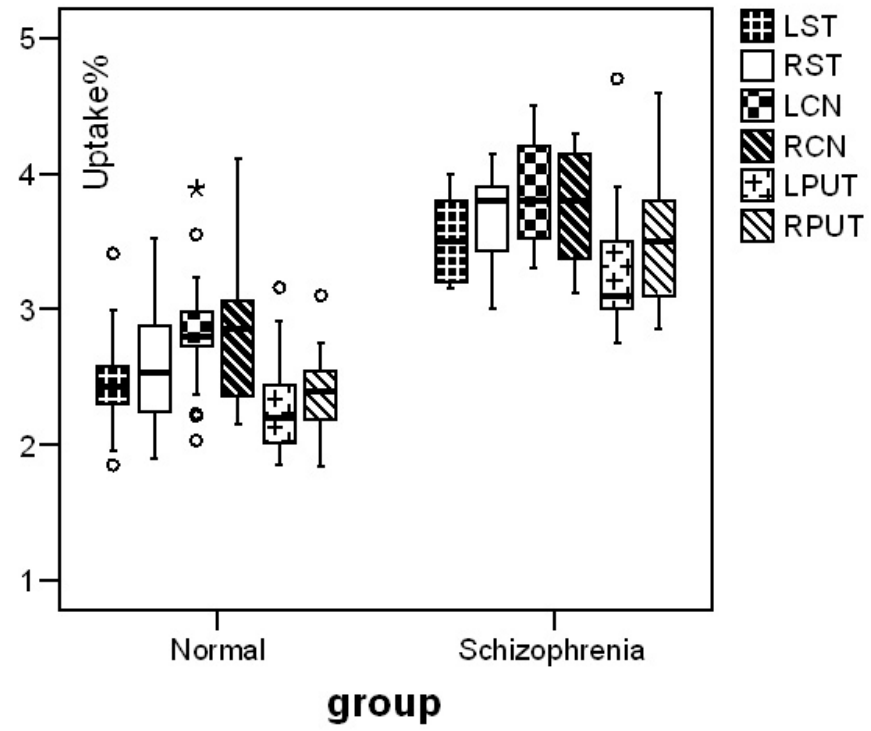

Figure 2. Median and inter-quartile range and outliers of radiotracer ( $\left.{ }^{123} \mathrm{I}-\mathrm{FP}-\mathrm{CIT}\right)$ uptake $\%$ in striatum, caudate nuclei and putamen in normal and schizophrenic patients

(Figure 3). No correlation with striatal uptake of the radiotracer and blood flow in these regions and also with age and gender was observed. Figure 4 shows MRI, HMPAO SPECT and DaT scan images of one of our patients with schizophrenia and DaT scan image in a normal agematched control. This image also shows that If DaT scan image was evaluated only visually it could not be differentiated from a normal image. Semiquantitative evaluation of the images in such cases is mandatory.

\section{Discussion}

Numerous studies have shown that dysregulation in neurotransmitter systems play a role in the pathophysiology of schizophrenia. Results of brain imaging studies with PET and SPECT in patients with schizophrenia have shown dysregulation of the dopaminergic system. Increased occupancy of $\mathrm{D} 2$ receptors by dopamine was demonstrated in studies with patients during the clinical exacerbation of the disease [13]. Additionally, increased dopamine in the synaptic cleft was predictive of good response to therapy of positive symptoms. The contributions of dopaminergic imbalance that underlay negative symptoms are not consistent. Although dopamine receptor abnormalities have been detected, this dysregulation seems to be a more complex process rather than simple abnormality in dopamine receptor number or expression. There might be abnormalities in receptor-interacting proteins of dopamine, particularly in transcript expression of proteins which are involved in dopamine signaling $[14,15]$. Seeman proposed the model of a common final pathway through hyperstimulation of D2 receptors that lead to psychosis by elevating the affinity status of D2 receptors [16].

We found an increase in striatal presynaptic dopamine transporters in our patients which may also reflect an increase in number of dopaminergic neurons in these patients. The patients with less positive symptoms showed more increased DaT in their caudate nuclei and right striatum. The negative correlation between the radiotracer uptake and positive symptoms of PANSS may show physiopathological polymorphism of the disease.

There are controversies with other authors. Lavalaye J et al. reported that striatal ${ }^{123}$ I-FP-CIT binding was not significantly different among antipsychotic-naive patients, those treated with olanzapine, patients treated with risperidone, antipsychotic-free patients and controls $(\mathrm{F}=0.07, \mathrm{p}=0.98)$ and also striatal ${ }^{123}$ I-FP-CIT binding in females was significantly higher than in males (3.29 and 2.70) and concluded that functional changes in the dopaminergic system in schizophrenia are not likely to be reflected in a change in DA transporter density [17]. Moreover, dopamine transporter density does not seem to be altered by antipsychotic medication. Mateos J et al. using DaT Scan, reported striatal dopamine transporter density decrease in first episode schizophrenia patients treated with risperidone [18]. Hsiao et al. in a study of 12 drug-naive schizophrenics with TRODAT-1 reported increase of presynaptic dopamine transporters in the left striatum but decrease in the right striatum [19]. Our patients showed a negative syndrome type. These controversies may be due to population group differences. Between-study heterogeneity as gender, age, PANSS type, gene polymorphism and small sample sizes may cause different findings in different studies. The event that presynaptic dopamine transporter (DaT) may play an important role as a regulator of synaptic dopamine concentration in schizophrenic patients should be considered and studied more.

It is discovered that the blockade of $60 \%$ to $80 \%$ of human cerebral dopamine D2 receptors is sufficient to reduce psychotic signs and symptoms in most cases with schizophrenia. Also, an elevation of $30 \%$ to $100 \%$ in the number of D2 receptors in post-mortem brains of schizophrenia cases, suggests that dopamine system may be overactive in schizophrenia [8,9]. This elevation of the total number of D2 receptors has been shown by Hirvonen et al. in healthy identical twins of schizophrenia individuals. They concluded that caudate dopamine D2 receptor up-regulation is related to genetic risk for schizophrenia. Higher dopamine D2 receptor density in caudate is also associated with poorer performance on cognitive tasks that involve corticostriatal pathways. This finding suggests that caudate dopamine dysregulation is also a trait phenomenon that contributes to psychosis vulnerability [20]. Kim et al. suggested that decreased nitration of tyrosine hydroxylase $(\mathrm{TH})$ that is present in patients with schizophrenia may be present in normal conditions also to limit the activity of this enzyme. Consequently, decreased nitration of TH in schizophrenia could act

We studied the blood flow in different brain regions also to find out if there is any correlation between blood flow in basal ganglia and dopamine transporter alteration. No difference in blood flow was observed in basal ganglia regions comparing controls and no correlation with striatal uptake of the radiopharmaceutical. We found a reduction of cerebral blood flow in bilateral prefrontal, occipital and right inferior parietal and temporal regions. Several studies have 

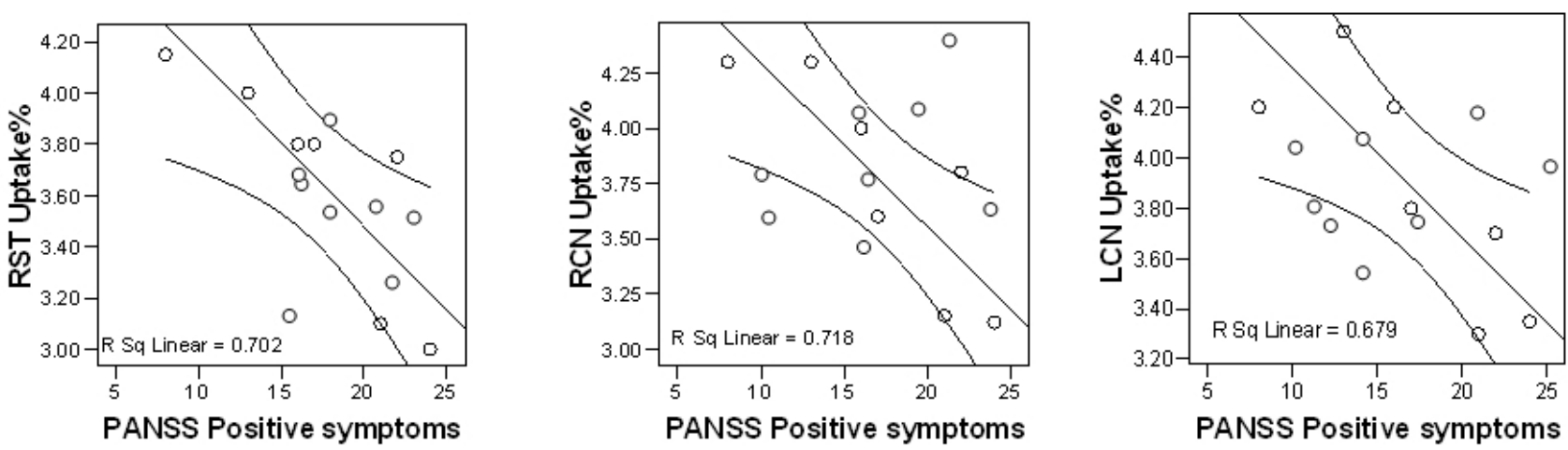

Figure 3. Shows the negative correlations of PANSS positive symptoms with radiotracer uptake\% in caudate nuclei and right striatum of our seven patients

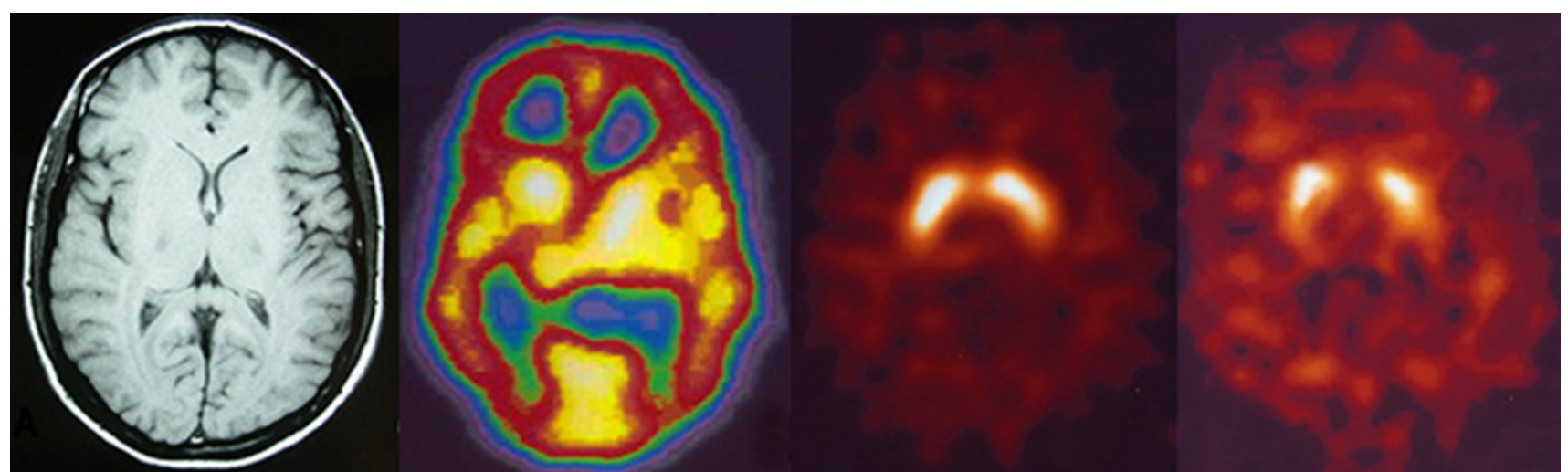

Figure 4. From left to right MRI, HMPAO SPECT and DaT scan images of a patient with schizophrenia. This patient showed increased radiotracer uptake in straitum. The forth scan shows DaT scan image in a normal age-matched control

reported different reduction of blood perfusion in schizophrenia. Cerebral perfusion reduction in different regions that we found should be studied and compared with our different psychometrics findings, which is not the objective of this study. Alterations in the prefrontal cortex are consistently implicated in psychiatric disorders, where studies have reported decreased metabolism, morphological changes [22] and increased oxidative damage [23]. Prefrontal cortex has a high relative density of dopaminergic projections compared with other cortical regions [24], and dopamine is involved in the regulation of working memory by the prefrontal cortex [25], which is impaired in patients with schizophrenia $[26,27]$.

In our study the patients are drug-naive which is an advantage that we believe can cover the low number of patients which is the limitation of our study.

\section{Conclusion}

First-episode drug-naive patients with predominant negative syndrome type have an increased striatal DaT binding assessed with ${ }^{123} \mathrm{I}$ FP-CIT. This increase in presynaptic dopamine transporter may be part of the neuronal abnormality which is associated with dopaminergic activity in schizophrenia and not secondary to the antipsychotic treatment or blood flow alterations. This study provides additional information about the involvement of the dopaminergic system in the pathophysiology of schizophrenia and the potential presence of a physiopatholological variation in two types of Schizophrenia.

Semiquantetive analysis of visually normal images of DaT scan in clinical practice seems mandatory in such cases.

\section{Disclosures}

Funding: No funder has supported this study.

Conflict of Interest: The authors declare that they have no competing interests.

Compliance with ethical standards: The research protocol was approved by the Ethical Committee of Neuro-psychiatric Department of Aristotle University of Thessaloniki.

\section{References}

1. Castle D, Wessely S, Der G, Murray RM (1991) The incidence of operationally defined schizophrenia in Camberwell, 1965-84. Br J Psychiatry 159: 790-794. [Crossref]

2. Bhugra D (2006) The global prevalence of schizophrenia. PLoS Medicine 5: 372-373.

3. Goldner EM, Hsu L, Waraich P, Somers JM (2002) Prevalence and incidence studies of schizophrenic disorders: a systematic review of the literature. Can J Psychiatry 47: 833-843. [Crossref]

4. Dutta R, Greene T, Addington J, McKenzie K, Phillips M, et al. (2007) Biological, life course, and cross-cultural studies all point toward the value of dimensional and developmental ratings in the classification of psychosis. Schizophr Bull 33: 868-876. [Crossref]

5. Meisenzahl EM, Schmitt JG. Scheuerecker J, Moller HJ (2007) The role of dopamine for the pathophysiology of schizophrenia. Int Rev Psychiatry 19: 337-345.

6. Cuesta MJ, Basterra V, Sanchez-Torres A, Peralta V (2009) Controversies surrounding the diagnosis of schizophrenia and other psychoses. Expert Rev Neurother 10: 14751486. [Crossref]

7. Sedaghat F, Gotzamani-Psarrakou A, Dedousi E, Arnaoutoglou M, Psarrakos K, et al. (2007) Evaluation of dopaminergic function in frontotemporal dementia using I-FPCIT single photon emission computed tomography. Neurodegener Dis 4: 382-385. [Crossref] 
8. Seeman P, Lee T, Chau-Wong M, Wong K (1976) Antipsychotic drug doses and neuroleptic/dopamine receptors. Nature 261: 717-719. [Crossref]

9. Lee T, Seeman P (1980) Elevation of brain neuroleptic/dopamine receptors in schizophrenia. Am J Psychiatry 137: 191-197. [Crossref]

10. Seeman P, Ulpian C, Bergeron C, Riederer P, Jellinger K, et al. (1984) Bimodal distribution of dopamine receptor densities in brains of schizophrenics. Science 225: 728-731. [Crossref]

11. Jibson MD, Tandon R (1998) New atypical antipsychotic medications. J Psychiatr Res 32: 215-228. [Crossref]

12. Kay SR, Fiszbein A, Opler LA (1987) The positive and negative syndrome scale (PANSS) for schizophrenia. Schizophr Bull 13: 261-276. [Crossref]

13. Abi-Dargham A, Rodenhiser J, Printz D, Zea-Ponce Y, Gil R, et al. (2000) Increased baseline occupancy of D2 receptors by dopamine in schizophrenia. Proc Natl Acad Sci US A 97: 8104-8109. [Crossref]

14. Koh PO, Bergson C, Undie AS, Goldman-Rakic PS, Lidow MS (2003) Up-regulation of the D1 dopamine receptor-interacting protein, calcyon, in patients with schizophrenia. Arch Gen Psychiatry 60: 311-319. [Crossref]

15. Baracskay KL, Haroutunian V, Meador-Woodruff JH (2006) Dopamine receptor signaling molecules are altered in elderly schizophrenic cortex. Synapse 15: 271-279. [Crossref]

16. Seeman P (2006) Targeting the dopamine D2 receptor in schizophrenia. Expert Opin Ther Targets 10: 515-531. [Crossref]

17. Lavalaye J, Linszen DH, Booij J, Dingemans PM, Reneman L, et al. (2001) Dopamine transporter density in young patients with schizophrenia assessed with [123] FP-CIT SPECT. Schizophr Res 15: 59-67. [Crossref]
18. Mateos JJ, Lomena F, Parellada E, Font M, Fernandez E, et al. (2006) Striatal dopamine transporter density decrease in first episode schizophrenic patients treated with risperidone. Rev Esp Med Nucl 25: 159-165. [Crossref]

19. Hsiao MC, Lin KJ, Liu CY, Tzen KY, Yen TC (2003) Dopamine transporter change in drug-naive schizophrenia: an imaging study with 99mTc-TRODAT-1. Schizophr Res 65: 39-46. [Crossref]

20. Hirvonen J, van Erp TG, Huttunen J, Aalto S, Nagren K, et al. (2005) Increased caudate dopamine D2 receptor availability as a genetic marker for schizophrenia. Arch Gen Psychiatry 62: 371-378. [Crossref]

21. Kim HK, Andreazza AC, Yeung PY, Isaacs-Trepanier C, Young LT (2014) Oxidation and nitration in dopaminergic areas of the prefrontal cortex from patients with bipolar disorder and schizophrenia. J Psychiatry Neurosci 39: 276-285. [Crossref]

22. Almeida JR, Akkal D, Hassel S (2009) Reduced gray matter volume in ventral prefrontal cortex but not amygdala in bipolar disorder: significant effects of gender and trait anxiety. Psychiatry Res 171: 54-68. [Crossref]

23. Andreazza AC, Shao L, Wang JF (2010) Mitochondrial complex I activity and oxidative damage to mitochondrial proteins in the prefrontal cortex of patients with bipolar disorder. Arch Gen Psychiatry 67: 360-368. [Crossref]

24. Ciliax BJ, Drash GW, Staley JK (1999) Immunocytochemical localization of the dopamine transporter in human brain. J Comp Neurol 409: 38-56. [Crossref]

25. D'Ardenne K, Eshel N, Luka J, Lenartowicz A, Nystrom LE, et al. (2012) Role of prefrontal cortex and the midbrain dopamine system in working memory updating. Proc Natl Acad Sci U S A 109: 19900-19909. [Crossref]

26. Glahn DC, Bearden CE, Cakir S (2006) Differential working memory impairment in bipolar disorder and schizophrenia: effects of lifetime history of psychosis. Bipolar Disord 8: 117-123. [Crossref]

27. Thompson JM, Gray JM, Hughes JH (2007) Impaired working memory monitoring in euthymic bipolar patients. Bipolar Disord 9: 478-489. [Crossref]

Copyright: (C2017 Sedaghat F. This is an open-access article distributed under the terms of the Creative Commons Attribution License, which permits unrestricted use, distribution, and reproduction in any medium, provided the original author and source are credited. 